\title{
Service Discovery and Provision for Autonomic Mobile Computing*
}

\author{
George C. Polyzos, Christopher N. Ververidis, and Elias C. Efstathiou \\ Mobile Multimedia Laboratory, Department of Computer Science, \\ Athens University of Economics and Business, \\ Athens 10434, Greece \\ \{polyzos, chris, efstath\}@aueb.gr
}

\begin{abstract}
Service discovery and related service advertisements, redirection and provision decisions are essential processes in networks supporting mobile communications in order for these systems to be self-configurable with zero or minimal administration overhead. More so in mobile networks, i.e. networks where the network infrastructure is moving and the topology is constantly changing. Finally, the servers themselves offering the services might be mobile, wirelessly connected and battery powered and thus power limited and energy constrained, with a finite horizon of operation and service availability. For this reason they will probably have rather selective policies for service advertisement and provision. In this paper we review our previous work on topics in this area and put it under this new perspective, providing our vision for a general autonomic framework for service advertisement, discovery, provision decision, redirection etc.
\end{abstract}

\section{Introduction}

The primary goal for Autonomic Systems is to enable systems to manage themselves given high-level objectives from administrators. Self-management of systems involves self-configuration, self-optimization, self-healing and self-protection. In mobile communications and pervasive environments a crucial facet of self-management is service discovery. If a flexible service discovery framework is lacking, nodes in a mobile, and in particular in infrastructure-less and constantly changing, environment will be confined to use only their own services and resources (or at most any such services and resources that are pre-configured by system administrators). Service discovery is thus paramount for operation in unknown environments (and for practical reasons in mobile and pervasive environments). Even though many service discovery protocols and architectures have been proposed, particularly for volatile environments such as those of Mobile Ad hoc NETworks (MANETs), none addresses all of the aforementioned aspects of self management and most are very far from being considered autonomic.

Autonomic service discovery is the gateway to autonomic mobile computing, since it enables heterogeneous nodes to interact with each other, learn and adapt to their

The original version of this chapter was revised: The copyright line was incorrect. This has been corrected. The Erratum to this chapter is available at DOI: 10.1007/ 978-3-540-32993-0_29

* Supported in part by IST FET ACCA coordination action. 
environment. Without this component no self-configuration, self-optimization, selfhealing or self-protection can be performed. For example, imagine a scenario where a node joins a MANET and initially has no knowledge of any resources located around it, neither does it host a well-known protocol for discovering services and resources. Up to now all the proposed approaches to service discovery assume that all nodes run the same discovery protocol, which may be very far from true in such heterogeneous environments, either due to the different capabilities of mobile hosts (e.g., differences of orders of magnitude in available local resources and performance metrics when comparing laptops to PDAs), or to their diversity (type of device and type of use and circumstances, e.g., slow or very fast movement). It is clear that in such a case nodes would not be able to demonstrate even the simplest autonomic behavior. What would change the situation in the above scenario would be a discovery approach allowing nodes to negotiate how to implement the discovery process. In this paper we shall discuss the need for a general framework for autonomic service advertisement, discovery, provision decision (on whether and to whom) in such networks along with our vision for the basic components of such a framework.

In our effort to introduce our vision of a universal autonomic framework (and also show the way towards its realization) we will present three ways for applying the concept of autonomy in service discovery and provision in different architectural contexts and over different network technologies. In order to realize autonomic systems we believe that we need to begin from service discovery, which itself should be inherently autonomic. Thus, first we will discuss how service discovery for MANETs may be enriched with autonomic properties. Then, we will provide an overview of a proposed architecture for global service publishing, discovery and access over a fixed infrastructure, where a critical contribution towards the autonomic goals is the use of ontologies for the description (and thus discovery) of services. As we will see in that section, this is a key idea for supporting really heterogeneous environments. In addition that architecture supports and emphasizes context and scheduling for service selection and provision. Finally, we will focus on service provision decisions. We have applied autonomic principles in the design of a fully decentralized system for service provision (with indirect reciprocity) in Wireless LANs (WLANs) with no central managing authority and even no strong (persistent, verifiable) identities.

\section{Autonomic Service Discovery}

Significant academic and industrial research has led to the development of a number of protocols, platforms and architectures for service discovery such as JINI [1], Salutation [2], UPnP [3], UDDI [4], Bluetooth's SDP [5] and SLP [6]. Most of these approaches were designed for static networks employing centralized approaches and implying reliable communication and enough bandwidth provided by the underlying networks. Recently newer approaches such as Allia [7], GSD [8], DEAPspace [9], Konark [10] and SANDMAN [11] were developed with pervasive computing environments in mind.

From our point of view a service discovery protocol or framework, especially for the harsh environment of MANETs, in order to be autonomic, should posses the following properties [12] or at least address the following issues: 
- Distributed-decentralized: This means that it should not rely on fixed or well known a priori infrastructure (e.g., a centralized service directory), but it should be able to discover services in a cooperative, on-demand, Peer-to-Peer (P2P) way. However, this does not exclude cases where specific powerful mobile nodes take up (temporarily) advanced roles, acting as service registries and allowing other potentially weaker nodes to publish and obtain services through them.

- Ontology-based: An autonomic service discovery framework should not rely on a priori knowledge of how services get to be known, but it should be able to semantically match services (with extensive use of ontologies). For example, two "mobile servers" may provide a similar service for currency conversion. However one of them may announce it as "currency conversion" and the other one as "currency exchange." So when a node is in need of a "currency conversion" service, by consulting the ontology, it should be able to identify both of these services as candidates for invocation.

- Context awareness for self-adaptation: The autonomic service discovery framework should be able to sense the environment by taking into account context information. This way service discovery can be self-adapted or self-configured based on high-level policies like "energy consumption minimization."

- Policy driven-election mechanism: Nodes should be free to choose the way service discovery is performed given their capabilities and goals. The service discovery framework should provide techniques to allow nodes to negotiate how service discovery will be performed. An election (majority) mechanism might be needed to decide on the preferred policy.

- Recovery mechanism: An autonomic service discovery should undoubtedly implement a "self-healing" process for recovering from service failures (e.g., due to a path break) after a node has invoked a service.

Our vision for autonomic service discovery points to a general framework responsible for disseminating the way that service discovery should be performed according to the administrator's goal. Since in MANETs there are no central (domain) administrators, this goal could be "translated" to being the result of the "common" goal of the users, for example "discovered" through an election performed by nodes participating in the MANET. In addition, different parts of the MANET could select different goals based on their local needs and requirements and hence tune the framework and its components to perform a different kind of service discovery in their area.

Our proposal is to split the service discovery into components, which can be tuned by every node according to their capabilities and policies. Hence, constrained nodes may select to use/participate in a more lightweight discovery process (e.g. directoryless and zone-limited), while more powerful and resource rich nodes may select advanced discovery policies. Important issues, such as aggregation and communication of information between neighboring areas with different goals and hence different ways to perform service discovery, are also addressed by the framework. A similar approach was proposed in [13] for autonomic routing. ${ }^{1}$

1 A “... programmable routing framework that creates an adaptable routing service for sensor networks. In this framework, a routing service is divided into several programmable components. Based on this division, a universal routing service is developed that allows the introduction of different services through a set of tunable parameters and programmable components." 
We also break service discovery into programmable components and allow tuning depending on application needs and management goals. The basic components are:

- Service advertisement (query vs. announcement, directory vs. directory-less, flooding vs. zone)

- Service selection (location based vs. energy conservation based vs. load based)

- Service recovery (statefull vs. stateless)

For example, a management goal could be: minimize total energy consumption and avoid draining a single server. This could be interpreted by the framework as: avoid using flooding, perform localized service discovery (e.g. up to 2 hops), implement pull techniques (i.e., queries from clients) for discovery and not push (i.e., advertisements from servers), encapsulate battery and current load information into service replies, automatically select closest server with minimal load etc.

Given the above approach, a high level of autonomy can be introduced into the nodes so that they can automatically cope with the increased levels of heterogeneity and volatility, which are present in a MANET environment. However many questions remain open. Among them:

- How do we select tunable components to include in the framework?

- How often can we change network or area-wide policies and goals?

- How can we allow and support different deployments of the service discovery approach in different areas?

- How can we aggregate information from neighboring areas with different goals and hence different ways to perform service discovery?

In the next paragraphs we continue discussing service discovery, but in a different context, that of a fixed infrastructure, highlighting the autonomic properties of a proposed architecture called Mobishare. We also discuss service publishing and optimized service access.

\section{An Architecture for Mobile Service Publishing, Discovery and Optimized Access over Fixed Infrastructures}

The rapid advances in wireless communications technology and mobile computing have enabled small, personal mobile devices that we use in everyday life to become information and service providers by complementing or replacing fixed-location servers connected to the wireline network. In the following we briefly describe the MobiShare architecture [14], which allows mobile servers to publish their services and mobile clients to discover and access these services over a distributed, possibly global, autonomic system, i.e., with no human intervention for low-level management. This system is capable of capturing context and uses it to self-optimize its responses when services are requested. Mobile servers publish their services through wireless networks to Administration Servers (ASs) that manage an area. These ASs are responsible for maintaining a list of services available (published) within their area of authority and also to provide a semantic discovery capability to assist the users with locating the services that can fulfill their needs by performing context-based filtering of query results. 
ASs are aware of and interconnected to other ASs over the (most probably fixed) network. Although they can function autonomously, in certain cases they may cooperate to perform inter-AS service discovery, mobile server positioning (for redirecting clients), and service migrations.

A major concern of an AS is to preserve service integrity throughout the system, since the same service description maybe available to more than one AS. So when publishing the service the user has to (1) declare an initial area where they wish the service to be available (user-defined policy) and (2) specify whether the service is fixed (i.e., an on-site service giving information about a monument) or mobile. In the case of a mobile service (i.e., a picture sharing service on the mobile "phone" of a tourist) the user (or its agent) must decide if the service availability area should be extended to the areas where the device moves (mobility-based policy) or to the areas where it is requested (request-based policy). This advertisement profile of a service is part of the service description and the ASs of the areas that publish the service are aware of it, since it determines whether they should proactively push the description to their neighbors. Every service description is initially stored to the AS to which the mobile server has published it for the first time. This AS is responsible for maintaining a list of all ASs to which the service may be propagated in the future. In this way whenever an update is issued to any AS which hosts the service description, this AS automatically obtains (from the original AS) the list of all ASs that store the service description and contacts them in order to update them too.

In order to minimize administrative and management overhead and provide a convenient level of autonomy when discovering and accessing services, a data-centric and services oriented approach is employed. Service providers publish their services to the infrastructure and service requestors discover them through the infrastructure. There is no human-to-human negotiation for publishing a service and the whole process is automated.

- Since a service is published there is no administrative overhead for managing it, but the system, according to the publication policy which is submitted along with the service, 'self-configures' it, determining the way that the service will be visible to requestors and to the rest of the system.

- For the discovery phase, the system's context sensing modules and semantic matching capabilities (with the help of an ontology), provide the system with 'self-optimization,' managing to keep bandwidth consumption low and user satisfaction high by returning only the most appropriate services to the user. (A non-optimized, not context-based, system would return a large number of candidate services, using excess bandwidth and overwhelming users with redundant and possibly useless information.)

- Also, in the case of a loss of a service provider, an AS may take up the role of executing the service (if at all possible), or alternatively finding another available service provider providing the same or a similar service, hence giving the system the so much anticipated 'self-healing' property.

Providing a convenient level of self configuration, self optimization and self healing the described architecture for service discovery, provision and access over fixed networks, shows the path towards realizing autonomy in such environments. Future research in this area could be in the direction of intelligent mechanisms for providing 
self protection to the system by automatically identifying and blocking malwareservices or possible DoS attacks.

In the next section we continue the presentation of our vision for autonomic computing by presenting a fully decentralized system that provides incentives and promotes cooperation of independent agents for service provision to peers.

\section{Autonomic Indirect Reciprocity-Based Peer-to-Peer Service}

The proliferation of low-cost networked digital devices enables new provisioning models for digital services. Traditional service models assume logically centralized providers, responding to service requests from authorized consumers. This general model covers a wide variety of network-based services ranging from basic Internet and content access to the provisioning of storage and computing services. This traditional model has several advantages. For example, the existence of a logically centralized authority, i.e. the service provider, which is usually assumed to be trustworthy, allows for simple solutions to common provisioning problems. From the provider's perspective, the main problem is the design of an appropriate charging model that would allow the provider to maximize profits. Traditional economic models can be used; for example, a utility-based mechanism design approach can assist in deriving the relevant parameters. Moreover, the existence of a centralized authority allows for the application of straightforward security solutions that protect the service from disruption and unauthorized usage. Finally, even though in many cases the service provider maintains a distributed network of service access points, this does not alter the basic "client-server" provisioning model. The physical distribution of services is usually adopted to address scalability and availability concerns. The physically distributed service is still under the control of a single authority, and there is usually a clean interface between the service's public and private layers. This physically distributed but logically centralized model encompasses, e.g., cellular operators and their networks of base stations, ISPs and their point-of-presence networks, and content providers and their networks of caches.

Recent research on what is generally referred to as the "peer-to-peer" (P2P) model of service provisioning revisits several of the assumptions above. Proposed P2P systems, such as multi-hop ad hoc and mesh networks, storage and file sharing networks, and grid computing networks, are all examples of a novel service provisioning model in which logically centralized (and profit-maximizing) authorities are absent. In the new model, the peers, in addition to consuming services, also take charge of service provisioning. The $\mathrm{P} 2 \mathrm{P}$ model is now becoming possible due to Moore's law, the drop in digital storage costs, the abundance of network bandwidth, and the existence of unlicensed wireless spectrum. Some of the most common P2P advantages cited include increased scalability and fault-tolerance, increased efficiency (in terms of pooling under-exploited resources), lower provisioning costs, and an increased sense of community-building among peers.

A less cited advantage of the P2P model, however, is the fact that it allows for near-zero-cost service configuration. To show how this is possible, in the following analysis we will assume a "pure" P2P system, i.e. a system in which centralized authorities are absent even during system initialization. In the traditional model, an important component of the overall cost is the cost incurred when consumers register 
with providers. This can include the cost of a provider-approved device, configured with the appropriate security credentials, or the administrative cost of an online or offline registration procedure that would provide the client with a unique system identity.

We will present a specific near-zero-cost configuration model that is applicable to a wide variety of pure P2P systems. In our previous work [15], we applied this model to a P2P wireless LAN roaming system of our design. The theoretical incentives issues behind this type of P2P model have been analyzed before by Feldman et al. [16].

Central to our model is the concept of a zero-cost system identity. Many P2P models assume a unique identity for every peer; they also assume that peers will not create more than one such identity (in order to avoid Sybil attacks ${ }^{2}$ [17], i.e., a no-cost change of identity). However, it is unclear how the "one peer, one identity" requirement can be met without a centralized trusted authority. Even though some designs acknowledge that this authority does not have to be online during the lifetime of the system, consumers are required, at the very least, to register with the authority when they first join the system.

In our registration-free model, identities are simple private-public key pairs. We assume that the private key is kept secret by the peer who wishes to adopt the specific identity, and that it is computationally infeasible to derive the private key from the digitally signed statements that are created with it. Obviously, peers can create multiple such identities if they so wish.

A peer's private key is used to sign a receipt each time service is consumed by a providing peer. Whenever such a transaction takes place (i.e. whenever a providing peer provides a service to a consuming peer), a receipt is generated and stored in the system. The receipt contains the public keys of the providing and consuming peer, a timestamp, and a "weight" which corresponds to the amount of service offered. The receipt is signed by the private key of the consuming peer. Our model assumes that all peers are selfish and rational and that peers will attempt to provide as few services as possible, while consuming as many services as possible. At the same time peers will attempt to hide any evidence of service consumption and forge evidence of service contribution. To increase the chances that the peers do actually sign receipts for the services they consume, the providing peers can request several (intermediate) receipts during service provisioning. This way, the risk of providing service and not obtaining the corresponding receipt can be minimized. The consumer has no choice but to sign whatever the provider requests in order for service provisioning to continue without interruption. If the consumer believes the provider is making unreasonable claims the only choice would be to abort the transaction. This, however, would cost the provider a useful receipt since receipts are to be used as evidence of service contribution.

The main idea behind the service model is to enable indirect reciprocity, a cooperation model that has been shown [16] to enable the evolution and stabilization of cooperation in self-organizing communities of selfish peers in the absence of authorities. To allow the evolution of cooperation, however, two additional issues need to be addressed. First, a peer's history must be visible to other peers. Second, collusion-based attacks that enable a peer to appear cooperative without really contributing to the

2 Sybil attack [17]: the creation of multiple identities per entity; a fundamental problem in open and self-organized electronically mediated communities without identity-certifying authorities. Sybil attacks can invalidate any number of system assumptions. 
community must be difficult or impossible to launch; the possibility of Sybil attacks (which are allowed in our model) makes the second task harder.

In [15] we presented a novel distributed history implementation that is incentivecompatible and allows peers to view subsets of the receipt graph and apply one of a family of collusion-resistant decision functions, which can guide peers in their provisioning decisions. Two main points that should underlie any distributed history implementation are the following. First, any gossiping algorithm that peers use in order to share their views of the receipt graph must take peer selfishness into account. It is safe to suggest that, irrespective of decision function, peers would want to hide their consumption actions and advertise only their contribution actions. Second, only shortterm history can be maintained. This practical limitation (finite storage capacity at the peers), however, works to the system's advantage: short-term history means that contributions and consumption actions that are in the past are erased from system memory. In an environment that relies on reciprocity this means that peers cannot rely on their previous contributions indefinitely, and that continuous cooperation is necessary.

A specific gossiping algorithm works as follows. Each peer maintains a local repository of receipts. The general replacement rule for this repository is "oldest one out," that is, when the repository is full, receipts with newer timestamps replace receipts with the oldest timestamps. This local repository certainly includes the receipts that have been directly "earned" by the peer when cooperating as provider (the consuming peers present these new receipts to the providers directly). However, these receipts represent only a subset of the receipt graph. We observe, however, that requesting peers have an incentive to show their own directly-earned receipts to their prospective providing peers (irrespective of specific decision function, as we mentioned above). If peers share receipts in this manner, after a few iterations each peer becomes aware of many more receipts than the ones it earned directly when acting as a provider.

\section{Conclusions}

This paper is motivated by the need for introducing autonomy in service discovery and provision architectures for mobile computing environments. With this in mind, we have reviewed new architectures we have recently proposed that are demonstrating some autonomic characteristics. Collectively, these architectures address selfconfiguration (or zero configuration), policy-based self-configuration and adaptation (nodes automatically adapt to a service discovery policy based on a high-level goal), self-optimization and self-healing. In figure 1 we illustrate the basic facilitators for inducing autonomy in service discovery and provision for different mobile environments as explained in the above paragraphs.

It is evident that context awareness and the use of ontologies are of paramount importance in both MANETs and more centralized architectures for enabling selfconfiguration, self-adaptation and self-optimization. Election mechanisms for deciding the preferred advertisement, discovery and recovery policies are also mandatory for MANETs so that autonomic service discovery can be performed taking into account the capabilities and needs of the nodes. Finally, indirect reciprocity mechanisms are useful in decentralized environments for allowing autonomic service provision without the need for central coordinating entities. 


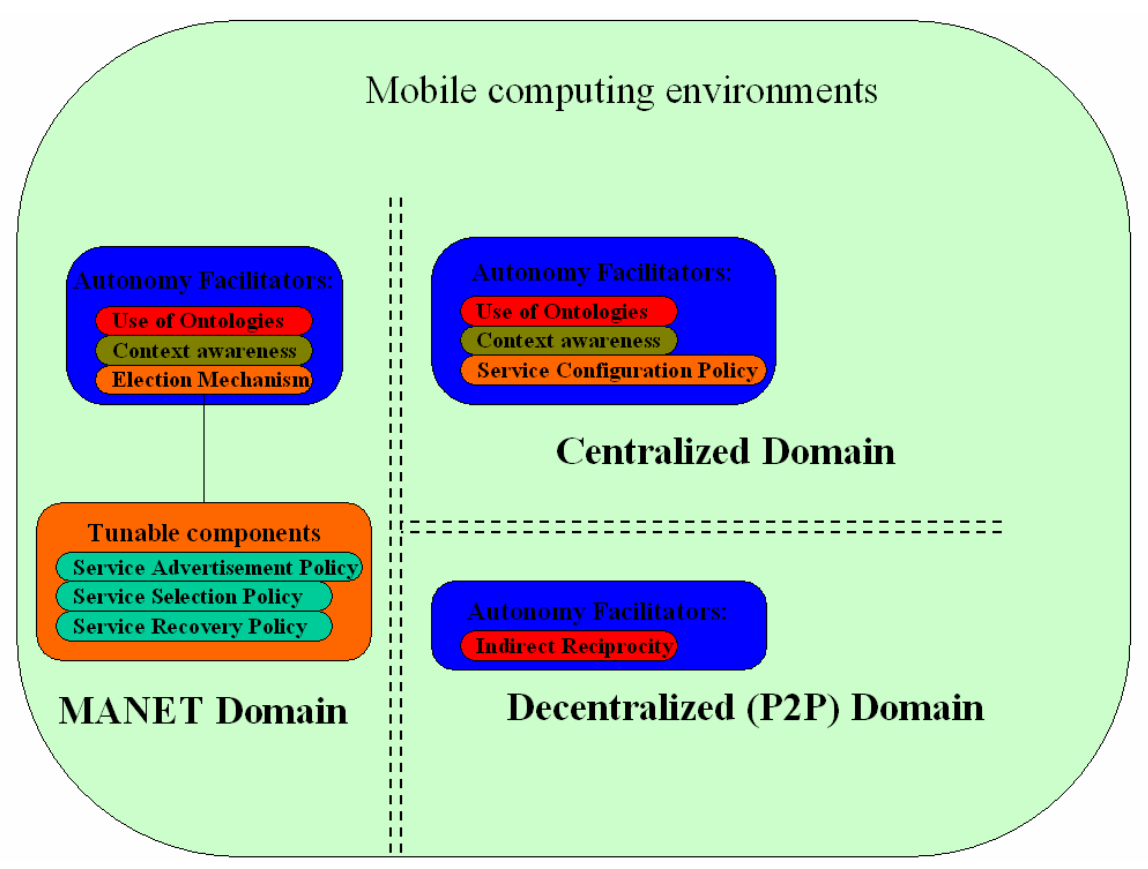

Fig. 1. Mobile Computing and Autonomy Facilitators for Service Discovery and Provision

At first glance there is no commonality between the autonomy facilitators presented across the different mobile environments. However, if we take a closer look, we can see that they can actually be merged under a common generic service discovery and provision framework suitable for addressing the autonomy needs of any mobile environment (see Figure 2).

To begin with the indirect reciprocity mechanisms as an autonomy facilitator, it is easy to understand that such mechanisms may be of great importance also in MANET environments. Especially if we take into account that service provision in MANETs is costly (nodes acting as providers or intermediaries expend valuable and scarce resources like energy and bandwidth) the network must provide a mechanism for enforcing fairness and ensuring cooperation between service providers and requestors. If this has to be done in a decentralized way without authorities, an indirect reciprocity mechanism, explained in previous paragraphs, is the perfect candidate. Also, in a centralized environment these mechanisms can be used in order for the system to implement autonomic self-protection by filtering out of the system malware services and nodes, based on reports/receipts published to the system by nodes that have used those services.

Finally, election mechanisms and tunable components as autonomy facilitators except for MANET environments can be proven useful also for centralized and decentralized environments for service provision. In the case of service provision, election mechanisms may be used to automatically decide the preferred provision policy and its rules. There is indeed much work yet to be done on realizing autonomic service 


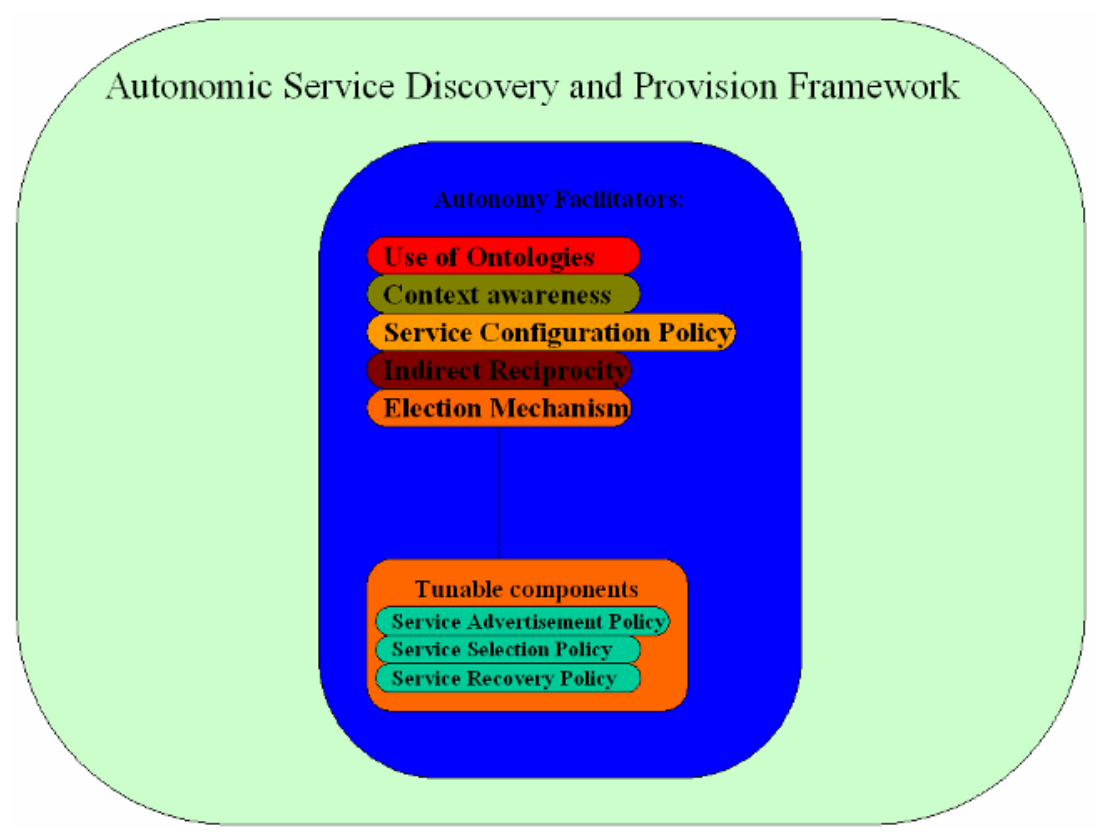

Fig. 2. Framework for Autonomic Service Discovery and Provision

discovery and provision for mobile computing, since until now research focused on the functional and optimization part of related architectures, instead of on their autonomic characteristics. We have identified the current lack of flexible-enough service discovery and provision protocols due to their monolithic nature and proposed, among others, breaking them into tunable components and implementing negotiation mechanisms among nodes (in order to select the preferred way of operation) as possible ways to increase their autonomy. This is certainly just the beginning of the investigation, but our proof of the existence of mechanisms for driving distributed systems with no central authorities and cheap identities towards evolutionary stability with near-zero configuration cost, is a step in the right direction. Towards this direction, we have tried to envision a generic framework by synthesizing autonomy facilitators, flexible enough for adding self-configuration, self-protection, self-healing and selfmanaging properties to any mobile computing environment dealing with service discovery and provision.

\section{References}

1. Sun Microsystems, "JINI Architecture Specification," November 1999.

2. Salutation Consortium, "Salutation Architecture Specification," 1999 (http://ww.salutation. org/specordr.htm).

3. Microsoft Corporation, "Universal Plug and Play: Background," 1999 (ww.upnp.org/resources/UPnPbkgnd.htm). 
4. "Universal Description Discovery and Integration Platform," September 2000 (http://www.uddi.org/pubs/Iru_UDDI_Technical_White_Paper.pdf).

5. "Specification of the Bluetooth System," December 1999 (http://www.bluetooth.com).

6. E. Guttman, C. Perkins, J. Veizades, and M. Day, "Service Location Protocol, Version 2," IETF RFC 2608, June 1999.

7. O. Ratsimor, D. Chakraborty, S. Tolia, D. Kushraj, A. Kunjithapatham, G. Gupta, A. Joshi, T. Finin, "Allia: Alliance-based Service Discovery for Ad-Hoc Environments," Proc. ACM Mobile Commerce Workshop, September 2002.

8. D. Chakraborty and A. Joshi, "GSD: A novel group-based service discovery protocol for MANETS", Proc. IEEE Conference on Mobile and Wireless Communications Networks, Stockholm, Sweden, September 2002.

9. M. Nidd, "Service Discovery in DEAPspace," IEEE Personal Communications, pp. 39-45, August 2001.

10. S. Helal, N. Desai, V. Verma, and C. Lee, "Konark - A Service Discovery and Delivery Protocol for Ad-Hoc Networks," Proc. 3rd IEEE Conference on Wireless Communication Networks (WCNC), New Orleans, Louisiana, March 2003.

11. G. Schiele, C. Becker and K. Rothermel, "Energy-Efficient Cluster-based Service Discovery for Ubiquitous Computing," Proc. 11th ACM SIGOPS European Workshop, Louven, Belgium, September 2004.

12. J.O. Kephart and D.M. Chess, "The Vision of Autonomic Computing," IEEE Computer, January 2003.

13. Y. He, C.S. Raghavendra, S. Berson, R. Braden, "A Programmable Routing Framework for Autonomic Sensor Networks," Proc. Autonomic Computing Workshop, Fifth Annual International Workshop on Active Middleware Services (AMS'03), Seattle, WA, pp. 60-68, June 2003.

14. E. Valavanis, C. Ververidis, M. Vazirgiannis, G.C. Polyzos, and K. Nørvåg, "MobiShare: Sharing Context-Dependent Data and Services from Mobile Sources," Proc. 2003 IEEE/WIC International Conference on Web Intelligence (WI 2003), Halifax, Canada, October 2003.

15. E.C. Efstathiou and G.C. Polyzos, "Self-Organized Peering of Wireless LAN Hotspots," European Transactions on Telecommunications, 2005 (special issue on Self-Organization in Mobile Networking, in press).

16. M. Feldman, K. Lai, I. Stoica, and J. Chuang, "Robust Incentive Techniques for Peer-toPeer Networks," Proc. 5th ACM Conference on Electronic Commerce, 2004.

17. J.R. Douceur, "The Sybil Attack," Proc. First International Workshop on Peer-to-Peer Systems (IPTPS'02), Cambridge, MA, 2002. 